C-A/AP/\#431

Sept. 2011

\title{
Analysis and correction of vertical dispersion in RHIC
}

\author{
C. Liu, Y. Luo, M. Minty \\ Brookhaven National Laboratory, Upton, NY 11973, USA
}

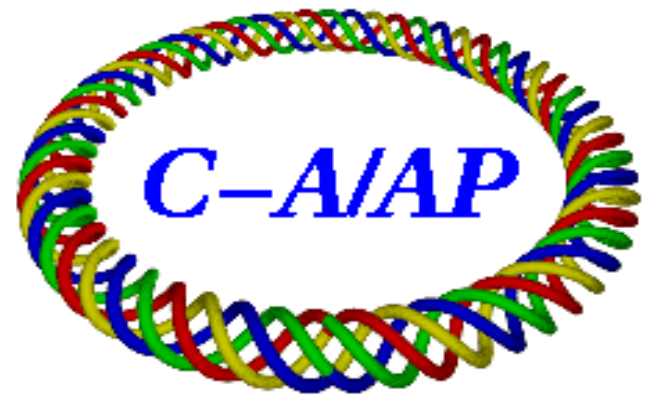

\section{Collider-Accelerator Department Brookhaven National Laboratory Upton, NY 11973}

Notice: This document has been authorized by employees of Brookhaven Science Associates, LLC under Contract No. DE-AC02-98CH10886 with the U.S. Department of Energy. The United States Government retains a nonexclusive, paid-up, irrevocable, world-wide license to publish or reproduce the published form of this document, or allow others to do so, for United States Government purposes. 


\title{
Analysis and correction of vertical dispersion in RHIC
}

\author{
C. Liu* and Y. Luo, M. Minty \\ BNL, Upton, NY, U.S.A. \\ (Dated: October 20, 2011)
}

\begin{abstract}
In the context of preserving the polarization of proton beams, the source of vertical dispersion in RHIC is analyzed. Contributions to dispersion from non-coupling sources and coupling sources are compared. Based on the analysis of sources for dispersion, the right actuator for correcting dispersion is determined and a corresponding algorithm is developed.
\end{abstract}

\section{THE MOTIVATION}

RHIC (Relativistic Heavy Ion Collider) is the only hadron collider with a high energy polarized proton physics program in the world. It consists of two rings (called Blue and Yellow) running in opposite direction. The figure of merit for physics experiment of polarized proton with longitudinal spin direction of both beam is $L \cdot P_{B l u e}^{2} \cdot P_{Y \text { ellow }}^{2}$ [1], where $L$ is the luminosity, and $P_{\text {blue }}$ and $P_{\text {yellow }}$ are the polarization of the Blue and Yellow beam respectively. Maintaining the polarization of both beams is a long-going effort in RHIC since there is $\sim 30 \%$ loss of absolute polarization from the source to average polarization over a physics store. A correlation between polarization and vertical dispersion has been discussed on various occassions and an effort of spin tracking with account of vertical dispersion is under consideration. Both vertical dispersion and depolarization may result from vertical deflections of the beam, which disturbs the spin axis to deviate from the vertical direction in a ideal machine. Therefore, correcting vertical dispersion should be able to help reducing the depolarizing source. How much improvement this would produce can only be answered by the forthcoming spin tracking effort or by implementation of a correction scheme as proposed in this report.

\section{SOURCE OF VERTICAL DISPERSION}

In this note, only vertical dispersion will be discussed. The source of vertical dispersion is divided into two categories (the reason for this division will be self-evident in later discusions):

- Non coupling contribution: vertical offset in quadrupoles..., dipole kick

- Coupling contribution: all coupling errors (quads roll, solenoids, snake alignment errors, offsets in sextuples, pitch of main dipole magnets, multipole errors...) and skew quadrupoles at locations with non-zero horizontal dispersion

As reasonable assumptions, the dispersion generated by beam offsets in quadrupoles is of the same order as that generated by the present settings of the vertical correctors, and dispersion generated by residual coupling sources (those not generated by skew quads) is of the same order of that from the presently used skew quadrupole settings.

Vertical dipole correctors and skew quadrupoles are two possible actuators that can be utilized to correct vertical dispersion. The choice between these two largely depends on how much dispersion can be generated by them compared with the residual dispersion in the machine. The following two sections are dedicated to answer this question.

\section{DISPERSION FROM CORRECTORS}

The differential equation for the vertical dispersion is (to first order, no coupling):

$$
\eta_{y}^{\prime \prime}+K \cdot \eta_{y}=\frac{1}{\rho},
$$

*cliu1@bnl.gov 
where, a prime indicates differentiation with respect to azimuthal location, $\eta_{y}$ is the vertical dispersion, $K$ is the local quadrupole strength, $\rho$ is the local bending radius.

Similar to closed orbit displacement, the solution for a point source of kick strength $\triangle y^{\prime}$ with periodic boundary condition is

$$
\eta_{y}=-\triangle y^{\prime} \cdot \frac{\sqrt{\beta_{y} \beta_{y 0}}}{2 \sin (\pi \cdot Q)} \cdot \cos \left(\pi \cdot Q-\left|\phi_{y 0}-\phi_{y}\right|\right)
$$

Here $\phi_{y 0}$ and $\phi_{y}$ are the betatron phase of the source point and reference point respectively; $\beta_{y 0}$ and $\beta_{y}$ are the betatron amplitude of source point and reference point; $Q$ is the vertical tune. Eq. (1) is exactly the same except of opposite sign as the solution for closed orbit displacements from a dipole error. The opposite sign is understandable because a positive closed orbit offset caused by a kick always produces a negative dispersion at the same point [2].

The beam offset in quadrupoles and sextupoles caused by the dipole kick will subsequently generate dispersion all along the ring, of which the effect has been discussed elsewhere [3, 4]. Here, the equation is quoted as

$$
M_{i j}=\frac{\sqrt{\beta_{i} \beta_{j}}}{2 \sin (\pi \cdot Q)}\left\{\begin{array}{cl}
-\cos \left(\pi \cdot Q-\left|\phi_{i}-\phi_{j}\right|\right) & \text { from dipole kick } \\
-\sum_{q} \frac{K 1}{2 \sin (\pi \cdot Q)} \beta_{q} \cos \left(\pi \cdot Q-\left|\phi_{i}-\phi_{q}\right|\right) \cos \left(\pi \cdot Q-\left|\phi_{q}-\phi_{j}\right|\right) & \text { from offset in quads } \\
+\sum_{s} \frac{\eta_{x} K 2}{2 \sin (\pi \cdot Q)} \beta_{s} \cos \left(\pi \cdot Q-\left|\phi_{i}-\phi_{s}\right|\right) \cos \left(\pi \cdot Q-\left|\phi_{s}-\phi_{j}\right|\right) & \text { from offset in sextupoles }
\end{array}\right.
$$

The subscripts $q$ and $s$ refer to quadrupole and sextupole respectively. This equation gives the response of vertical disperion to a vertical kick, the dispersion $\left(\eta_{x}\right)$ used in the eqation is horizontal dispersion. Therefore, the response of dispersion to corrector strength can be expressed in a matrix form,

$$
\left(\begin{array}{c}
D_{1} \\
D_{2} \\
\vdots \\
D_{m}
\end{array}\right)=\left(\begin{array}{cccc}
M_{11} & M_{12} & \cdots & M_{1 n} \\
M_{21} & M_{22} & \cdots & M_{2 n} \\
\vdots & \vdots & \ddots & \vdots \\
M_{m 1} & M_{m 2} & \cdots & M_{m n}
\end{array}\right) *\left(\begin{array}{c}
\theta_{1} \\
\theta_{2} \\
\vdots \\
\theta_{n}
\end{array}\right)
$$

Here $D_{i}$ is the dispersion at $i$ th BPM, $\theta_{j}$ is the kick strength of the $j$ th corrector.

Since we seek to compare the order of magnitude of coupling and noncoupling contribution to dispersion, the exact calculation of dispersion response is not necessary [5]. The following calculation in the note is based on pp11v7::store optics, dispersion measurement on Mar. 15, 2011 in blue ring. The dispersion at all BPMs from dipole kicks are calculated based on Eq. 2 and displayed in Fig. 1 together with the measured dispersion.

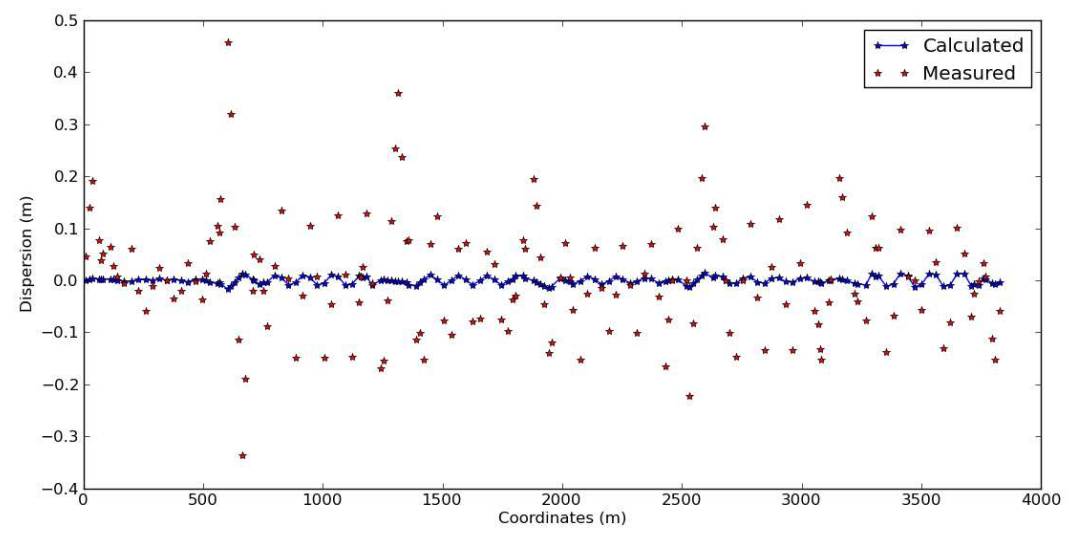

FIG. 1. Measured vertical dispersion vs. calculated dispersion generated by correctors

As we can see, the dispersion generated by vertical correctors is one order of magnitude lower than the measurement. Together with the assumption that dispersion generated from offsets in quadrupoles and from corrector kicks are of the same order, we come to the conclusion that this non-coupling factor is not the major contribution, and vertical correctors along will not be able to correct the measured vertical dispersion. 


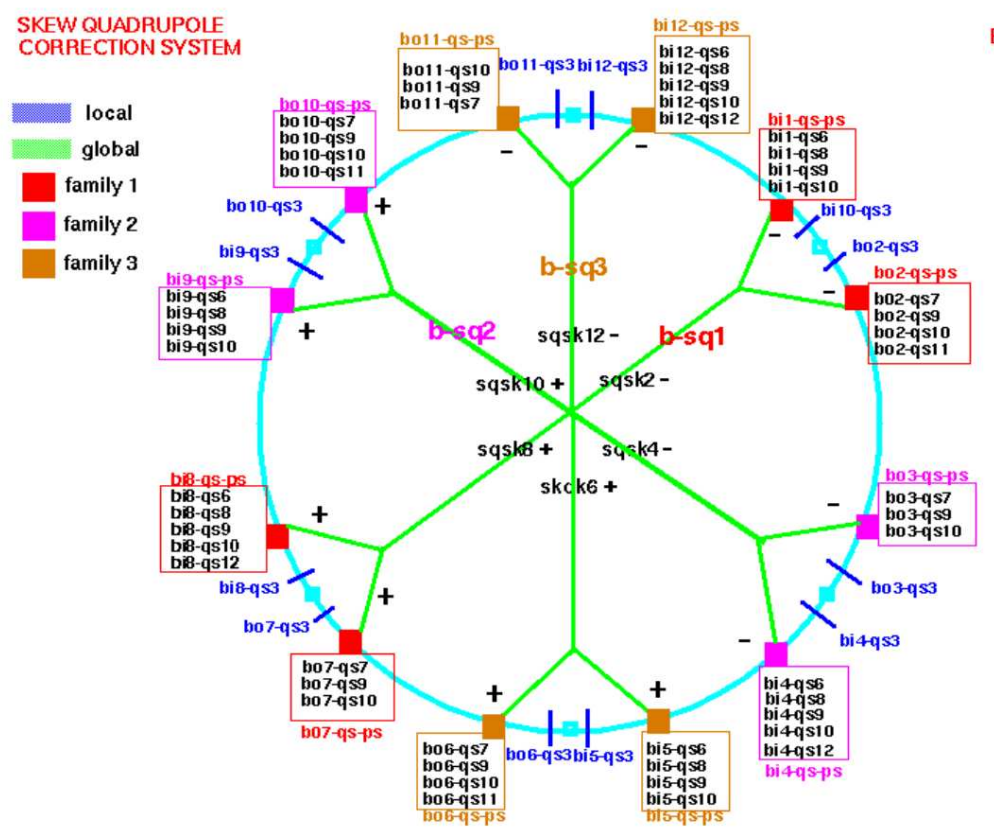

FIG. 2. SQ families configuration in the blue ring [6]

\section{DISPERSION FROM SKEW QUADRUPOLES}

The configuration of skew quads in the Blue and Yellow ring are similar. In Fig. 2 the schematic of skew quads in Blue is shown [6]. Skew quads in the interaction regions are used for correcting local coupling from triplet roll [7]. There is one skew quad for each triplet (total 12). Skew quads (total 48) between the arc and interaction region are for correcting linear difference global coupling [8], which are grouped as three families according to their coupling contribution's direction. There are 12 power supplies for these 48 skew quads, of which those in the same colored box have one common power supply. In the following, we break the cyan line running from one IR to its opposite IR to form a 6 -familiy configuration, and further break the cyan line running from one side of a IR to the other side to form a 12-familiy configuration.

The differential equation for vertical dispersion with coupling (to first order, corrector kick neglected) is

$$
\eta_{y}^{\prime \prime}+K \cdot \eta_{y}=(2 \theta k+S) \eta_{x}
$$

which assumes quadrupole rolls and skew quadrupoles as major coupling contributors, $\theta$ is the roll angle of a normal quadrupole, $S$ is the skew quad strength and $\eta_{x}$ is the horizontal dispersion at the coupling source point. The self-consistent solution [9] of dispersion when a skew quad strength changes by $\triangle(k l)$ is

$$
\triangle \eta_{y}=-\triangle(k l) \eta_{x} \cdot \frac{\sqrt{\beta_{y} \beta_{y 0}}}{2 \sin (\pi \cdot Q)} \cdot \cos \left(\pi \cdot Q-\left|\phi_{y 0}-\phi_{y}\right|\right)
$$

The response of dispersion to a unit change in skew quad strength can be expressed in matrix form as

$$
\left(\begin{array}{c}
D_{1} \\
D_{2} \\
\vdots \\
D_{m}
\end{array}\right)=\left(\begin{array}{cccc}
R_{11} & R_{12} & \cdots & R_{1 n} \\
R_{21} & R_{22} & \cdots & R_{2 n} \\
\vdots & \vdots & \ddots & \vdots \\
R_{m 1} & R_{m 2} & \cdots & R_{m n}
\end{array}\right) *\left(\begin{array}{c}
k_{1} \\
k_{2} \\
\vdots \\
k_{n}
\end{array}\right)
$$

where $D_{i}$ is the dispersion at $i$ th BPM, $k_{j}$ is the integral strength of the $j$ th skew quad, and $R_{i j}=-\frac{\sqrt{\beta_{i} \beta_{j}}}{2 \sin (\pi \cdot Q)} \eta_{x}$. $\cos \left(\pi \cdot Q-\left|\phi_{i}-\phi_{j}\right|\right)$.

Using the skew quads strength from the pp11v7::store optics, the dispersion at all BPMs generated by the 48 skew quads was calculated and displayed in Fig. 3 together with the measured dispersion. The dispersion generated by the 


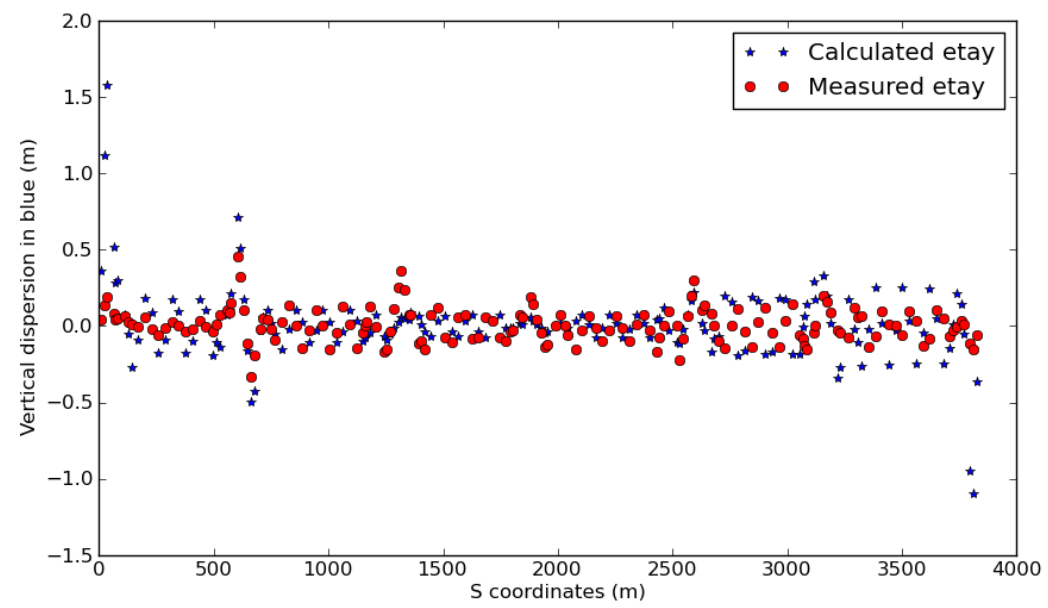

FIG. 3. Measured vertical dispersion vs. dispersion generated by those skew quadrupoles located between the arc and the IR (e.g. neglecting those skew quads used to compensate locally the effect of triplet roll)

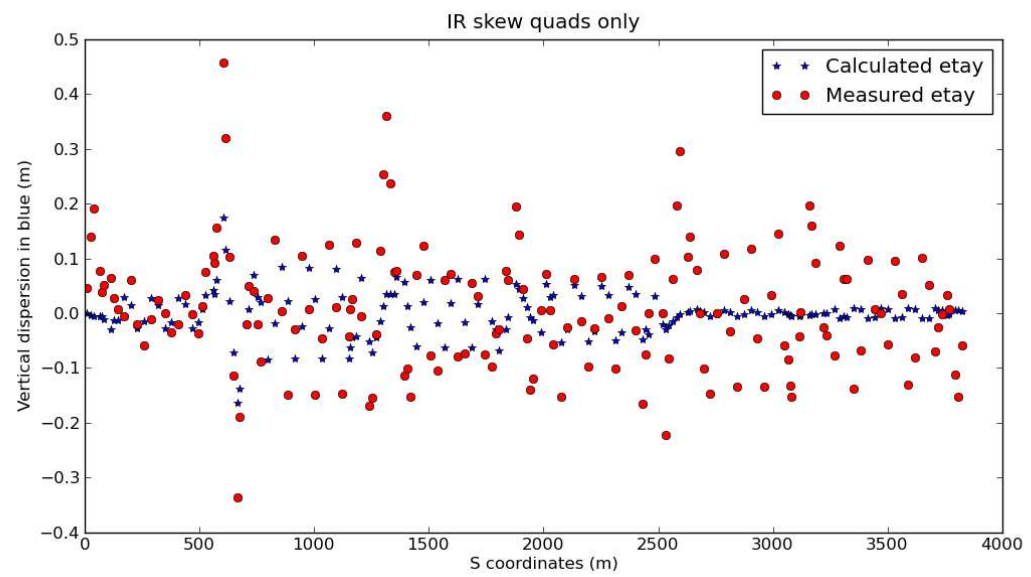

FIG. 4. Measured vertical dispersion vs. dispersion generated by IR SQs alone (e.g. neglecting those skew quads used to compensate linear global difference coupling)

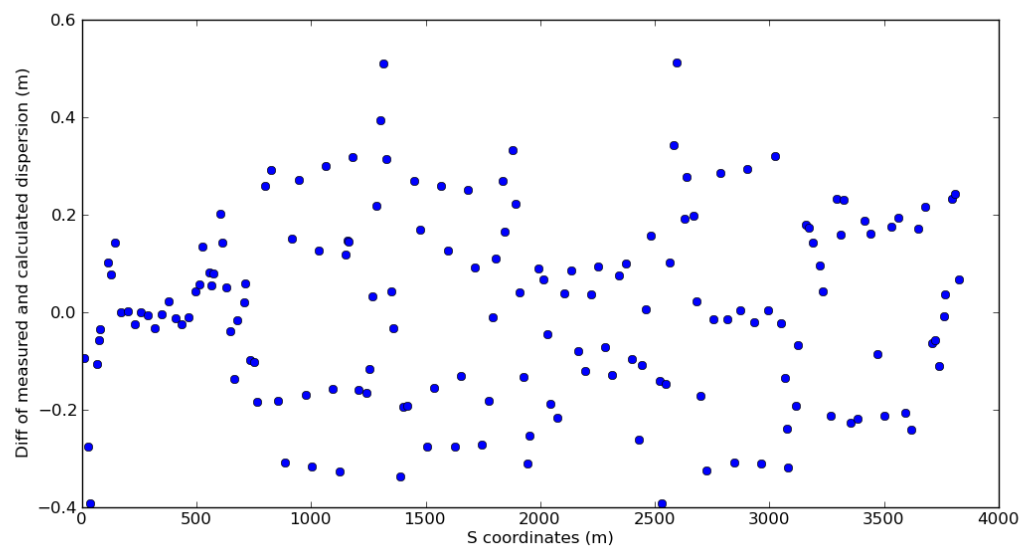

FIG. 5. The difference between measured dispersion and dispersion generated by all skew quads (e.g. dispersion from all coupling sources except skew quads) 
IR skew quads alone is also shown in Fig. 4 together with dispersion measurement; The difference between measured dispersion and dispersion generated by all skew quads is shown in Fig. 5 .

The conclusions from Figs. 3, 4 and 5 are: (1) coupling sources as a group is the dominating contributor to the vertical dispersion; (2) dispersion from coupling error (everything except skew quads), skew quads in between arc and IR, and skew quads in IR are of the same order, which justify the assumption in section II. Therefore, skew quads in between arc and IR will be the natural choice for correcting vertical dispersion.

As a confirmation of the analytical methods used, the dispersion generated by a strength change of $0.0011 / \mathrm{m}$ of one of the 6 skew quad families ( 8 skew quads around 6 o'clock) was calculated numerically as well [10]. This agrees very well with the analytical results as shown in Fig. 6.

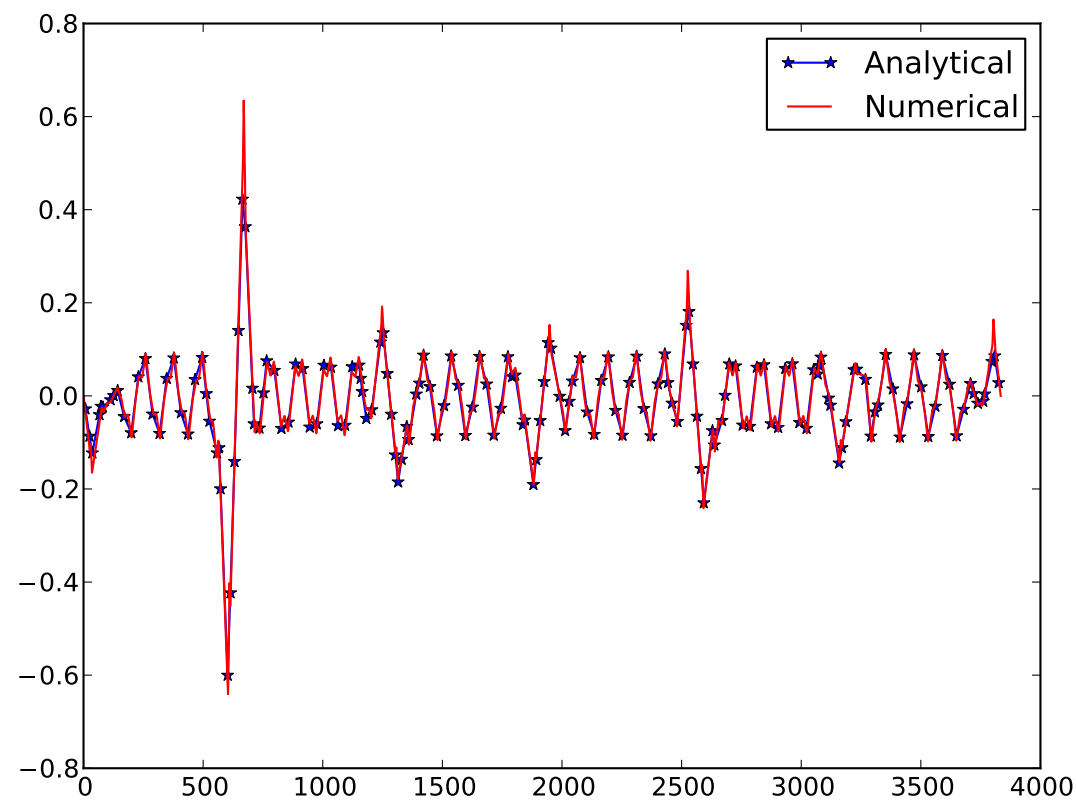

FIG. 6. Comparison of dispersion at BPMs in blue generated from first SQ family (6 o'clock, $k l=0.0011 / m)$ by numerical and analytical calculation

\section{DISPERSION CORRECTION}

With the measured dispersion at BPMs along the ring denoted as $\vec{D}_{0}=\left(\begin{array}{c}D_{1} \\ D_{2} \\ \vdots \\ D_{m}\end{array}\right)$, the goal of dispersion correction is $\vec{D}_{0}+\vec{D}_{s q}=0$, here $\vec{D}_{s q}$ is dispersion compensation generated by skew quads. Therefore, the required skew quads strength comply with a set of linear equations

$$
\vec{D}_{s q}=-\left(\begin{array}{c}
D_{1} \\
D_{2} \\
\vdots \\
D_{m}
\end{array}\right)=\left(\begin{array}{cccc}
R_{11} & R_{12} & \cdots & R_{1 n} \\
R_{21} & R_{22} & \cdots & R_{2 n} \\
\vdots & \vdots & \ddots & \vdots \\
R_{m 1} & R_{m 2} & \cdots & R_{m n}
\end{array}\right) *\left(\begin{array}{c}
k_{1} \\
k_{2} \\
\vdots \\
k_{n}
\end{array}\right)
$$

There are different algorithm for solving the above equations to get the required skew strength, here we adopt Singular Value Decomposition (SVD). 


\section{A. Correction with 3 SQ families}

Eq. 5 is the basis for the general algorithm for dispersion correction. If we use 3 families for the correction, a corresponding reduced $m \times 3$ matrix can be derived by manipulating the $m \times 48$ matrix which characterize the response of dispersion to 48 single skew quads. The correction results are shown in Fig. 7 and 8 . The original strengths correspond to paired skew quads strength (0 and 3,1 and 4,2 and 5) as presently used in the accelerator for correcting linear global coupling.

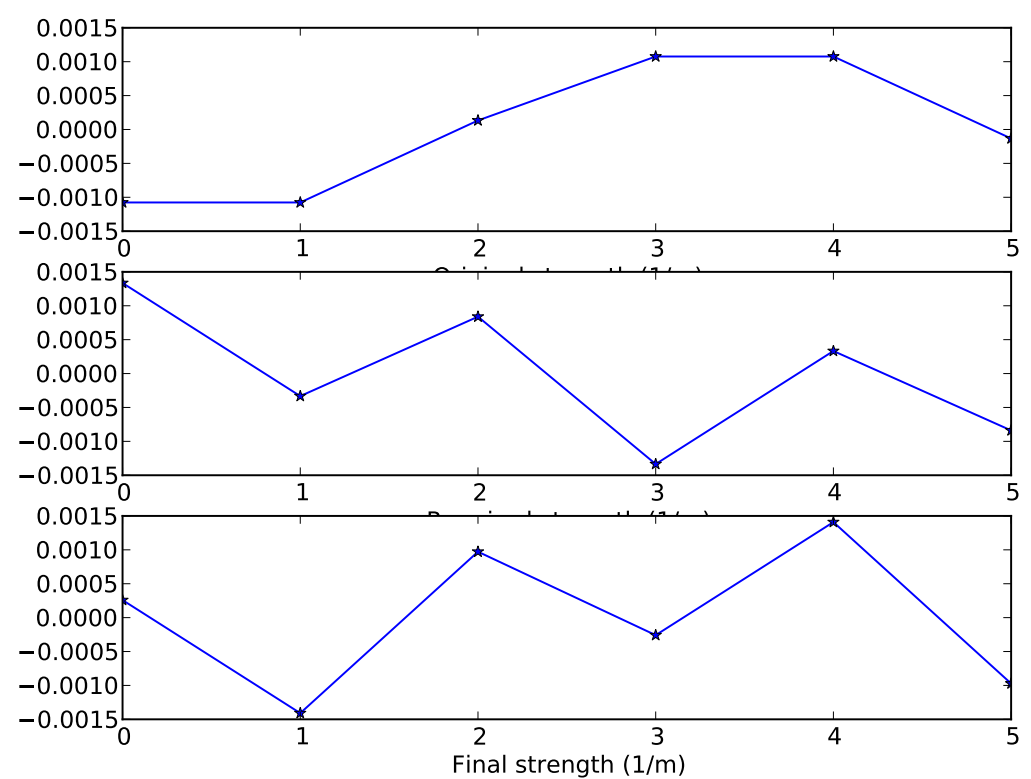

FIG. 7. Original (upper), required (middle) and final skew quads strength (lower) with 3 skew quads families
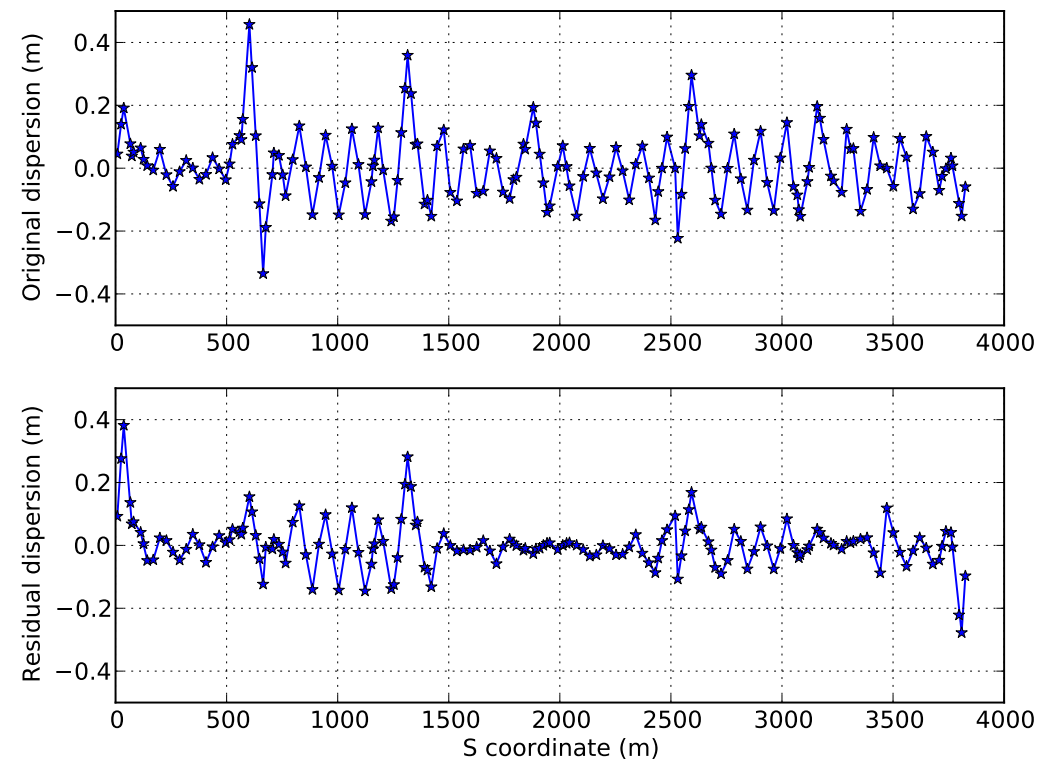

FIG. 8. Measured dispersion (upper) and final dispersion after correction (lower) with 3 skew quads families 


\section{B. Correction with 6 SQ families}

An $m \times 6$ matrix is generated in this scheme. The correction results are shown in Fig. 9 and 10 . More than three times smaller dispersion can be achieved by the 6-family correction scheme.

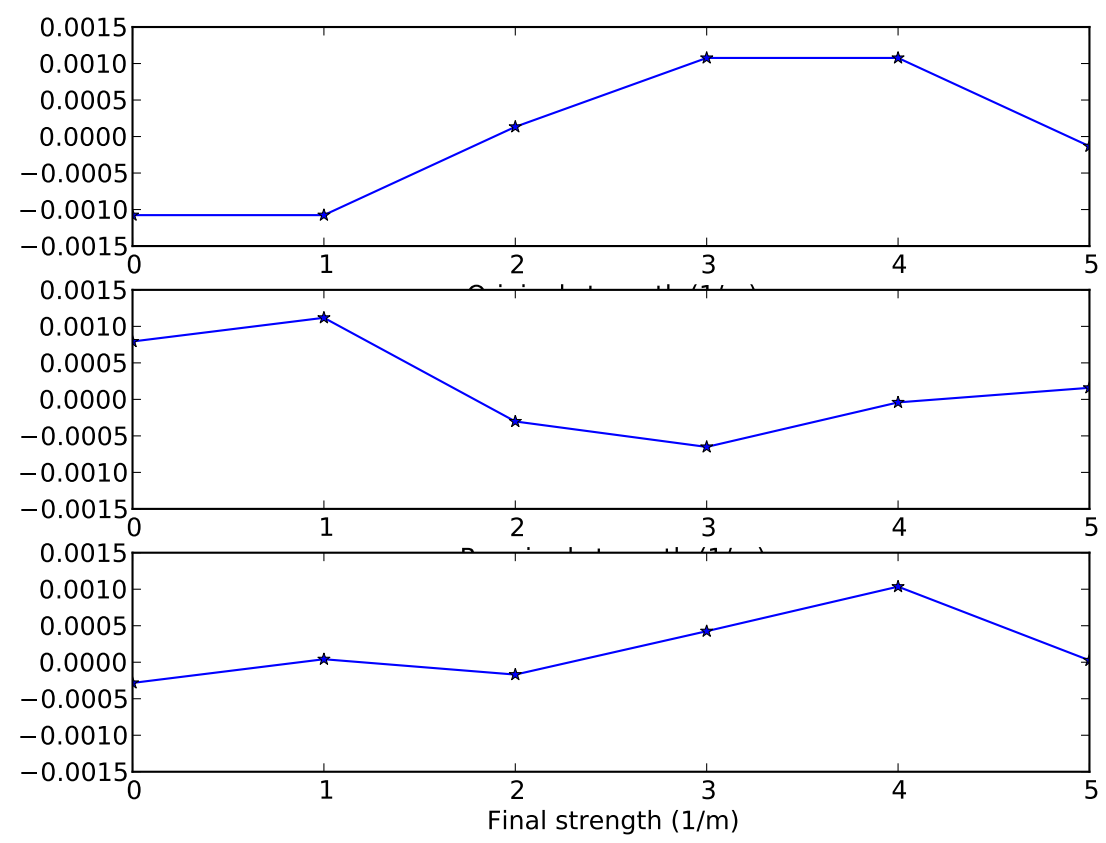

FIG. 9. Original, required and final skew quads strength with 6 skew quads families
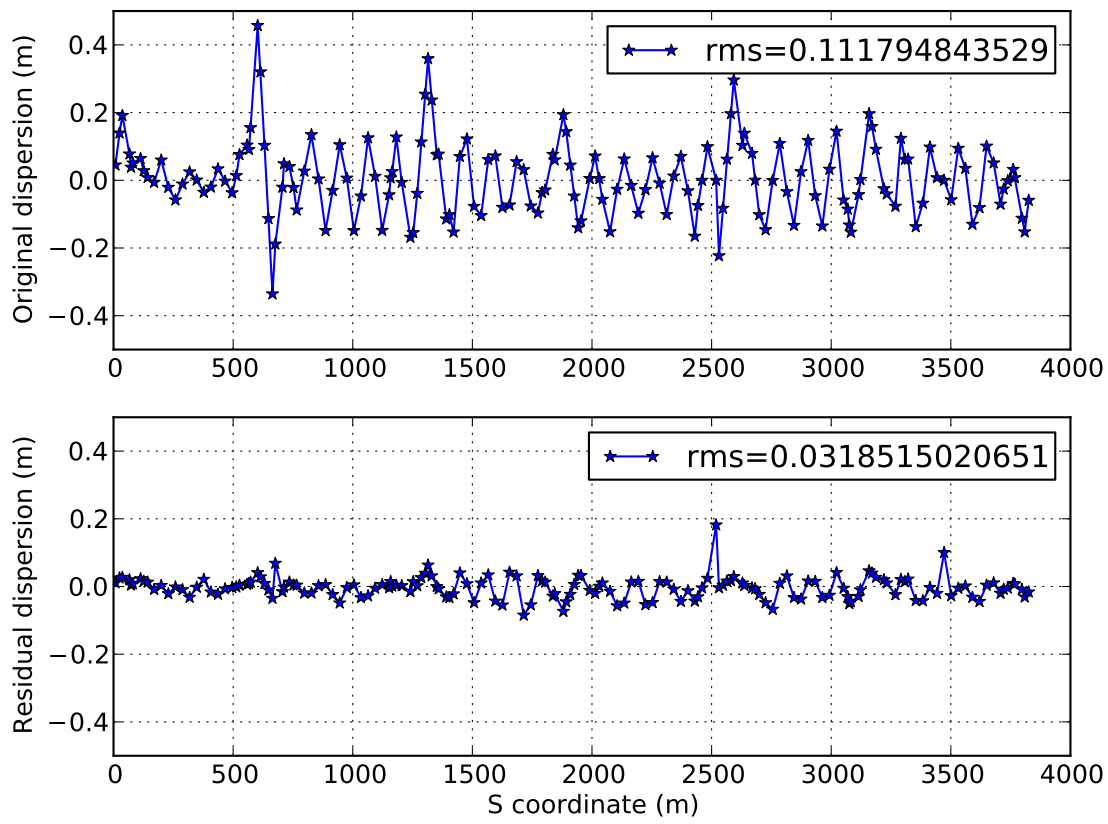

FIG. 10. Dispersion before and after correction with 6 skew quads families 


\section{IMPACT ON COUPLING}

Before our discussion of dispersion correction, the functionality of skew quads is only for correcting coupling. Although it has been demonstrated in section $\mathrm{V}$ that dispersion correction is feasible using skew quads families, further study of its impact on coupling is mandatory.

The linear difference coupling coefficient $C^{-}[8]$ generated by skew quads is given by

$$
C^{-}=\frac{1}{2 \pi} \sum_{i} \sqrt{\beta_{i x} \beta_{i y}} k_{i} l_{i} \exp \left(i\left(\phi_{i x}-\phi_{i y}-2 \pi \Delta \cdot s_{i} / L\right)\right) .
$$

Here $\beta_{i x, i y}$ are the uncoupled betatron amplitude functions, $\phi_{i x, i y}$ are the uncoupled betatron phase advances, $k_{i}$ and $l_{i}$ are individual skew quadrupole strength and length, $L$ is the ring circumference, and $s_{i}$ is the distance between the skew quadruple and the reference point. Based on Eq. 6, a matrix describing the respone of linear difference coupling coefficient to skew quads strength for the 6-family case is given by

$$
\left(\begin{array}{l}
\operatorname{Re}\left(C^{-}\right) \\
\operatorname{Im}\left(C^{-}\right)
\end{array}\right)=\left(\begin{array}{llllll}
N_{11} & N_{12} & N_{13} & N_{14} & N_{15} & N_{16} \\
N_{21} & N_{22} & N_{23} & N_{24} & N_{25} & N_{26}
\end{array}\right) *\left(\begin{array}{l}
k_{1} \\
k_{2} \\
k_{3} \\
k_{4} \\
k_{5} \\
k_{6}
\end{array}\right)
$$

In RHIC, the linear coupling is continuously measured by a Phase-Locked-Loop (PLL) tune meter. The residual coupling coefficient is derived from 4 measurables from the PLL [8],

$$
\begin{gathered}
R_{I}=\frac{\beta_{y}}{\beta_{x}} \cdot \frac{\left|C^{-}\right|}{\sqrt{\left(\triangle^{2}+\left|C^{-}\right|^{2}\right)}+\triangle} \\
R_{I I}=\frac{\beta_{x}}{\beta_{y}} \cdot \frac{\left|C^{-}\right|}{\sqrt{\left(\triangle^{2}+\left|C^{-}\right|^{2}\right)}+\triangle} \\
\triangle \phi_{I}=\chi \\
\triangle \phi_{I I}=\pi-\chi
\end{gathered}
$$

Here $R_{I}, R_{I I}$ are the ratios of the horizontal and vertical eigenmode projection amplitudes, $\triangle \phi_{I}$ and $\triangle \phi_{I I}$ are the eigenmode phase differences between the horizontal and vertical projections, and $\triangle$ is the fractional uncoupled tune split.

\section{ALGORITHM OF SIMULTANEOUS COUPLING AND DISPERSION CORRECTION}

In order to correct coupling and dispersion simultaneously, one needs to solve linear equations for both coupling and dispersion. The tricky part is to scale (e.g. effectively weight) the two sets of equations properly. The matrix form for a simultaneouly coupling and dispersion correction for the 6 -family case is

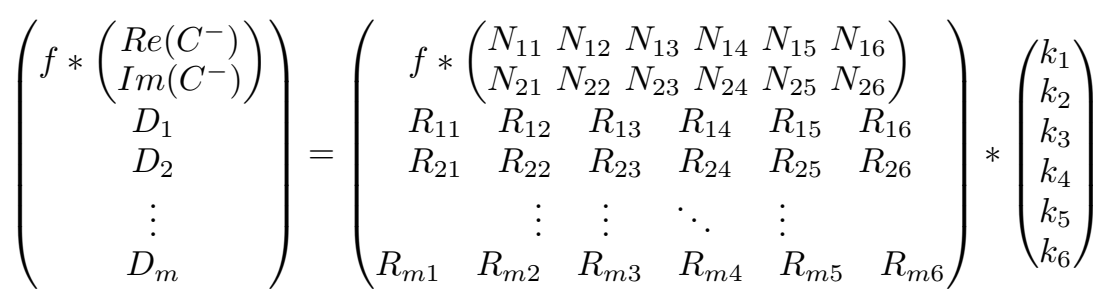

Here, $f$ is the scale factor, which weights the preference for coupling and dispersion correction.

For the correction algorithms, we start with a machine state in which all skew quads strength are zero. Although this state may not be real, it is straightforward and convenient for calculating the corrections. We call coupling in the machine except the contribution from skew quads $C_{\text {error }}$, and dispersion in the machine except contribution from skew quads $D_{\text {error }}$. Then it is true in a machine with linear coupling being compensated by skew quads

$$
\begin{gathered}
C_{\text {error }}+C_{s q} \sim 0 \\
D_{\text {error }}+D_{s q}=D_{\text {measured }}
\end{gathered}
$$




\section{SIMULTANEOUS DISPERSION AND COUPLING CORRECTION WITH 6-FAMILY SKEW QUADS}

In this section, we will discuss two cases: coupling correction with limited dispersion contribution from skew quads; and reduction of residual coupling and vertical dispersion at the same time. In the second case, coupling correction is weighted more heavily since any degradation of coupling correction is undesirable. The acceptable coupling coefficient $\left|C^{-}\right|$is taken as $10^{-4}[11]$.

\section{A. case I}

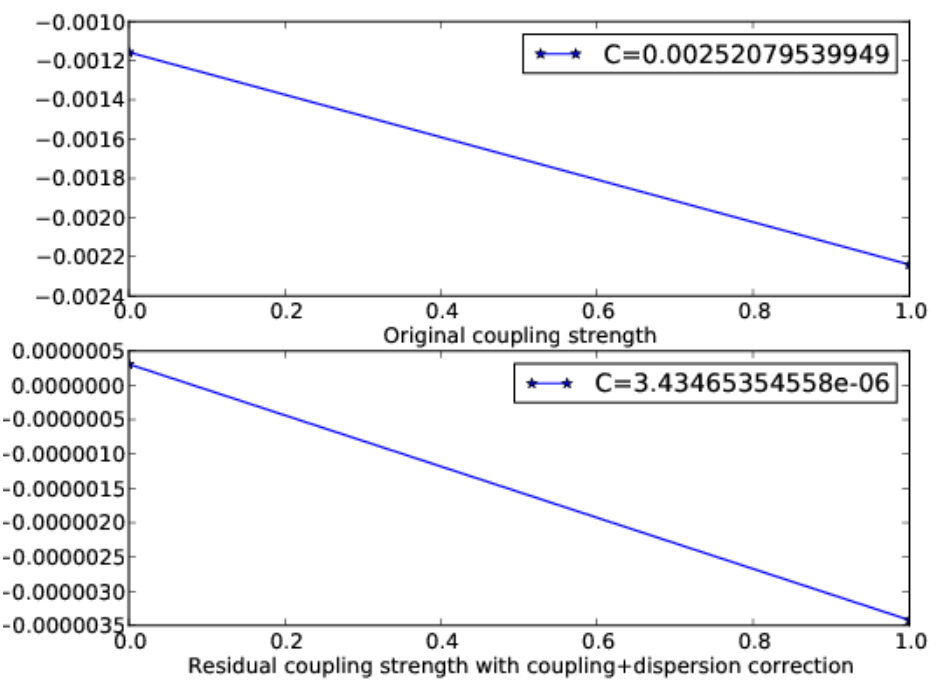

FIG. 11. Coupling before (upper) and after (lower) correction in case I (correcting global coupling with SQs and minimizing dispersion contribution from them) with 6 independent skew quads families

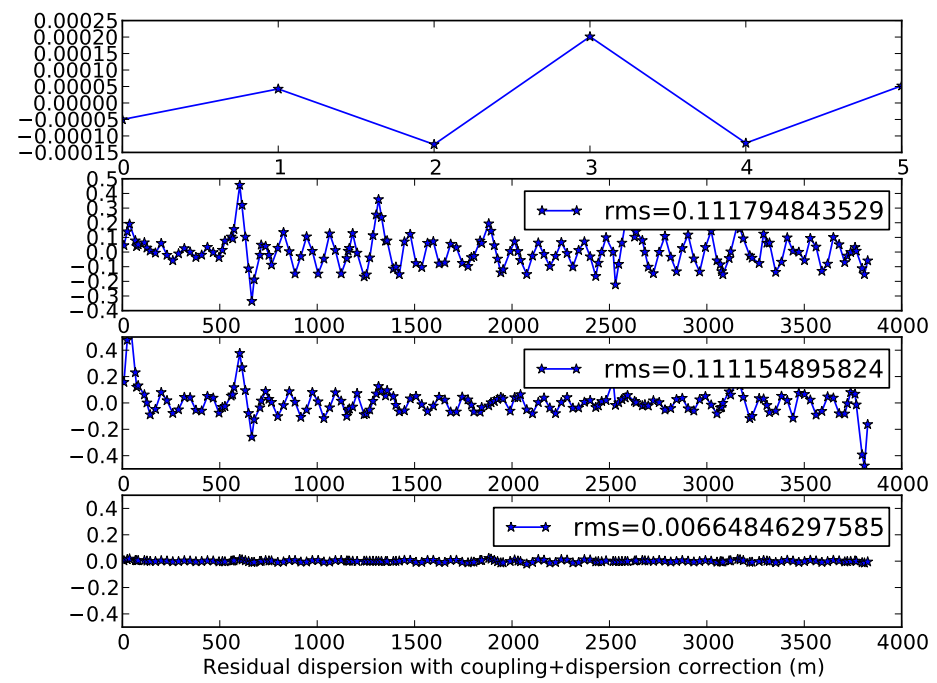

FIG. 12. Required strength (upper), measured dispersion (second), measured dispersion minus skew quads contribution (third) and introduced dispersion (lower) by correction in case I with 6 independent skew quads families 
In this case, our goal is

$$
\begin{gathered}
C_{\text {error }}\left(=-C_{s q}\right)+C_{\text {sq,new }} \longrightarrow 0 \\
D_{\text {error }}\left(=D_{\text {measured }}-D_{s q}\right)+D_{\text {sq,new }} \longrightarrow D_{\text {error }}
\end{gathered}
$$

$C_{s q, n e w}$ and $D_{s q, n e w}$ are the coupling and dispersion contributed by skew quads in the new correction scheme. By applying new strength to skew quads, one tries to cancel the global coupling with little contribution to the vertical dispersion. The goal is equivalent to setting all dispersion terms on the left side of Eq. 10 to zero. With a scale factor of 0.001, the results are shown in Fig. 11 and 12. From the upper plot in Fig. 11, the presently used skew quads strength can be reduced dramatically (by a factor of 5) while maintaining an adequate coupling correction by separating the existing 3 families to 6 . The second and third plot are the measured dispersion and dispersion of the machine after skew quads contribution being subtracted respectively. The dispersion introduced by the skew quads in the lower plot in Fig. 12 is controllable by the scale factor $f$.

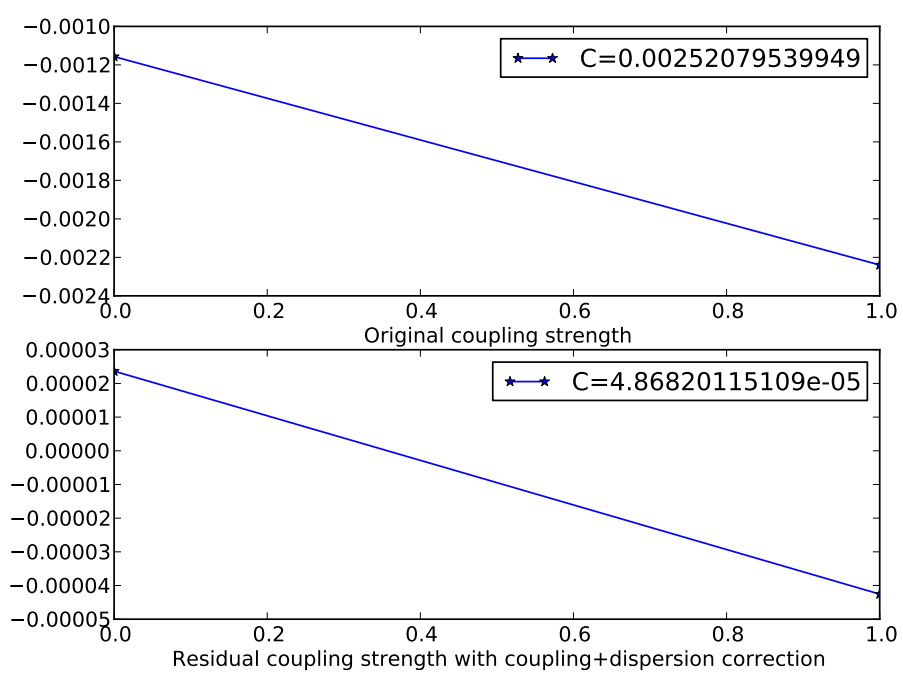

FIG. 13. Coupling before (upper) and after (lower) correction in case II

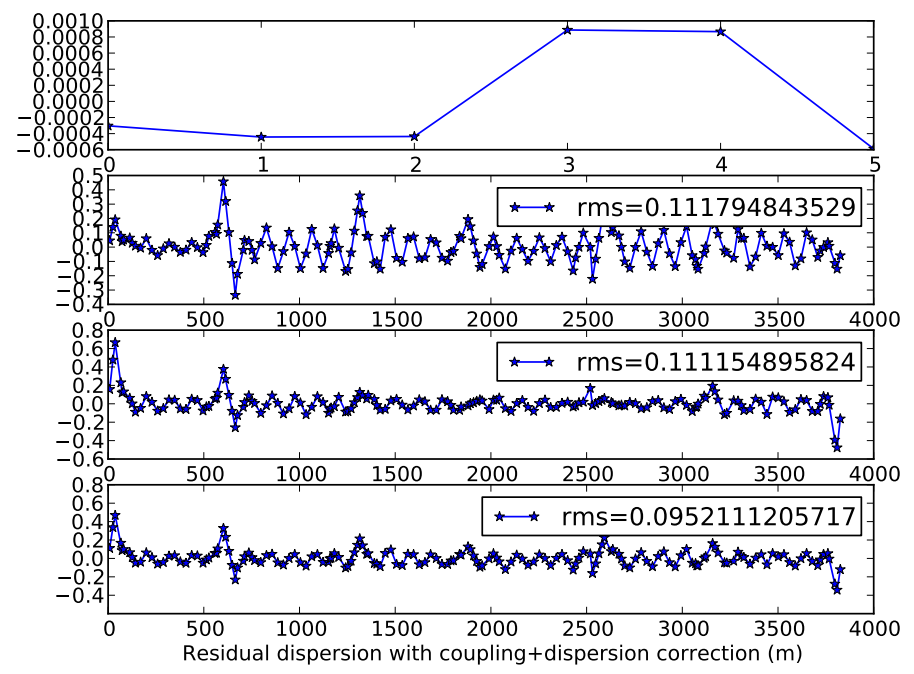

FIG. 14. Required strength (upper), measured dispersion (second), measured dispersion minus skew quads contribution (third) and final dispersion (lower) in case II with 6 independent skew quads families 


\section{B. case II}

The goal here is

$$
\begin{gathered}
C_{\text {error }}\left(=-C_{s q}\right)+C_{s q, \text { new }} \longrightarrow 0 \\
D_{\text {error }}\left(=D_{\text {measured }}-D_{s q}\right)+D_{\text {sq,new }} \longrightarrow 0
\end{gathered}
$$

By applying new strengths to skew quads, one tries to cancel both global coupling and vertical dispersion. The results are shown in Fig. 13 and 14. Although the coupling is kept below $10^{-4}$, the reduction of dispersion RMS $(\sim 15 \%)$ is not substantial to justify this new scheme.

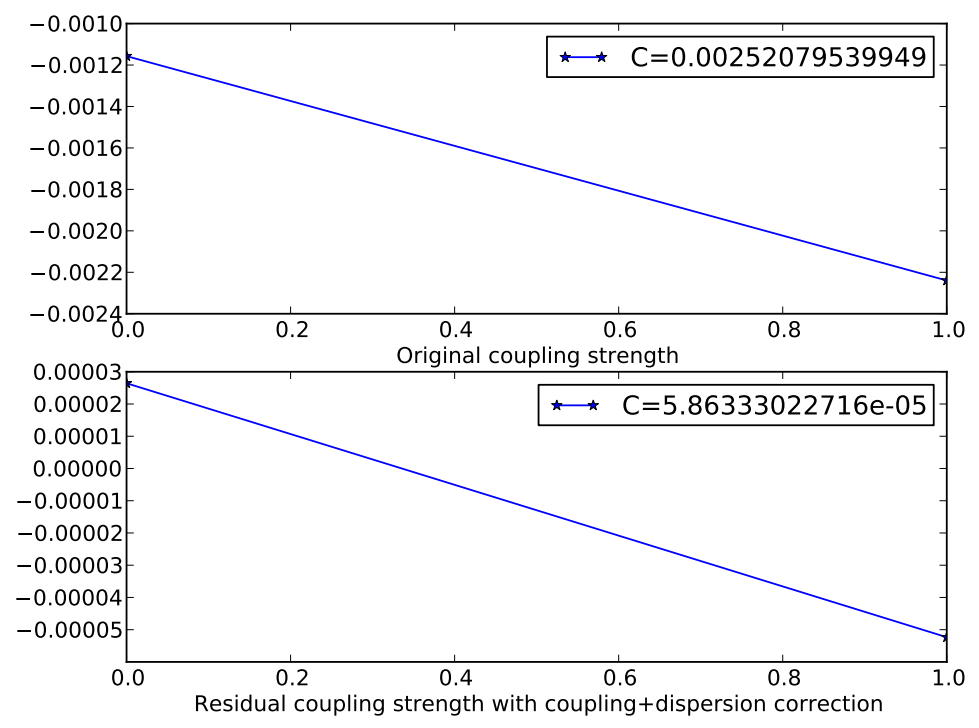

FIG. 15. Coupling before (upper) and after (lower) correction with 12 skew quads families

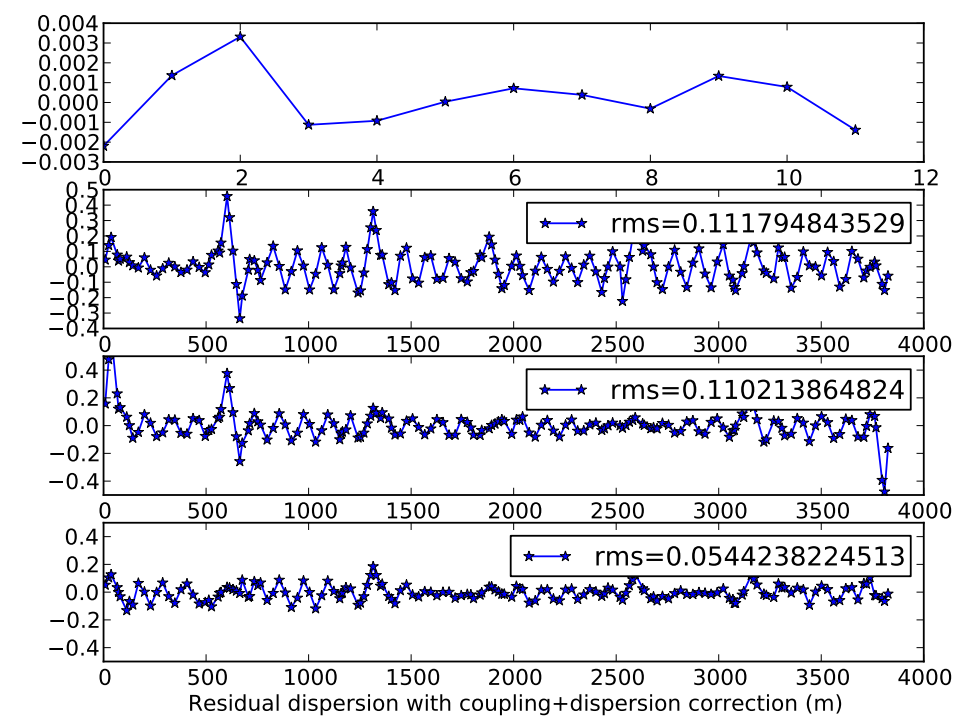

FIG. 16. Required strength (upper), measured dispersion (second), measured dispersion minus skew quads contribution (third) and final dispersion (lower) with correction by 12 skew quads families 


\section{SIMULTANEOUS CORRECTION WITH 12-FAMILY SKEW QUADS}

As mentioned in section IV, the 48 skew quads are actually on 12 power supplies. The performance of correction is improved with more degrees of freedom as expected. The coupling could be reduced by $\sim 50$ (Fig. 15), with a factor of 2 reduction of the dispersion (Fig. 16).

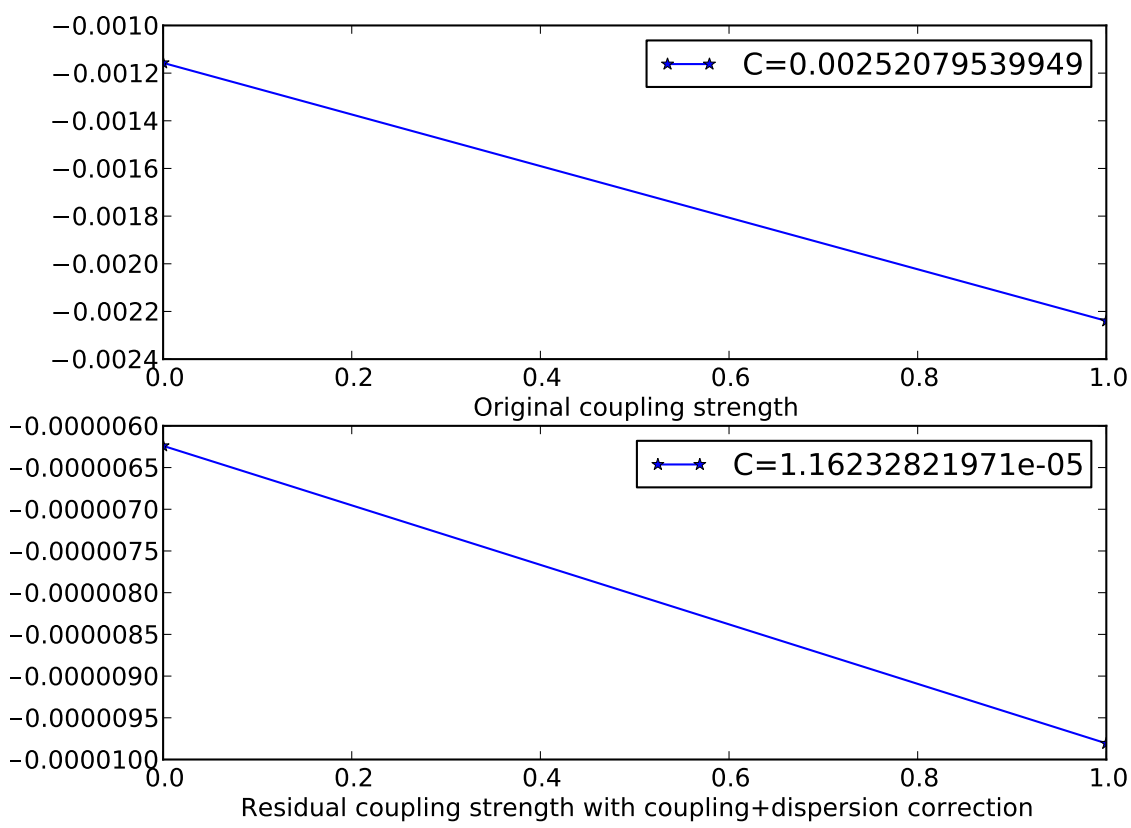

FIG. 17. Coupling before (top) and after (bottom) correction with 48 skew quads

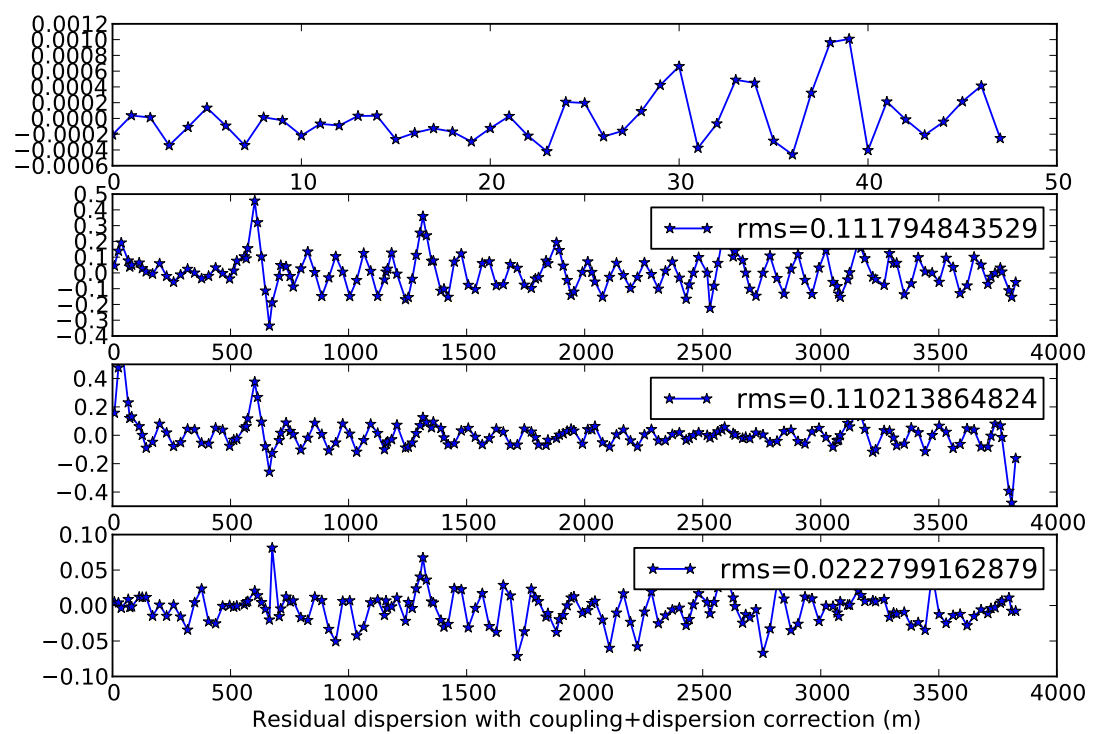

FIG. 18. Required strength (upper), measured dispersion (second), measured dispersion minus skew quads contribution (third) and final dispersion (lower) with correction by 48 skew quads 


\section{SIMULTANEOUS CORRECTION WITH 48 SKEW QUADS}

The calculation in this section is for consideration only given the significant effort that would be required for controlling the 48 skew quads independently. Shown in Figs. 17 and 18 are the coupling and dispersion before and after correction respectively. The residual disperison after correction with 48 skew quads is on the same order of magnitude as the dispersion that vertical correctors capable of generating. This means that vertical correctors could be used to further reduce the residual dispersion.

\section{CONCLUSION}

From analysis of the various sources for vertical dispersion, we showed that the vertical dispersion generated by coupling sources is $\sim 10$ times that generated by non-coupling sources (vertical dipole correctors). Therefore, skew quads in RHIC are chosen for correcting vertical dispersion. New schemes of correcting coupling and dispersion were proposed. One could reconfigure the skew quads families to reduce its strength by a factor of 5 while maintaining adequate coupling correction; or reduce dispersion by $\sim 15 \%$ while maintaining factor of 50 reduction of coupling with simultaneous correction by 6 families; or reduce dispersion by half while maintaining a factor of 50 reduction of coupling with simultaneous correction by 12 families; or even reduce dispersion by factor of 5 while maintaining a factor of 50 reduction of coupling with simultaneous correction by 48 skew quads. The change of coupling coefficient and RMS of the vertical dispersion for various correction schemes in blue ring are summarized in Table I, where $\left|C_{o}^{-}\right|$ and $\left|C_{f}^{-}\right|$are the original and the final coupling coefficient, $\eta_{r m s}^{o}$ and $\eta_{r m s}^{f}$ are the original and final vertical dispersion RMS, $\triangle \eta_{r m s}$ is the difference of them.

TABLE I. The change of coupling coefficient and RMS of the vertical dispersion for various correction schemes in blue ring

\begin{tabular}{|c|c|c|c|c|}
\hline Correction schemes & $\left|C_{f}^{-}\right|$ & $\left|C_{o}^{-}\right| /\left|C_{f}^{-}\right|$ & $\eta_{r m s}^{f}$ & $\Delta \eta_{r m s} / \eta_{r m s}^{o}$ \\
\hline 6-family (Case I) & $3.43 \mathrm{e}-6$ & 734.69 & $\sim 0.111$ & $\sim 0$ \\
\hline 6-family (Case II) & $4.87 \mathrm{e}-5$ & 51.75 & 0.095 & $14.4 \%$ \\
\hline 12-family & $5.86 \mathrm{e}-5$ & 43.00 & 0.054 & $51.0 \%$ \\
\hline 48 skew quads & $1.16 \mathrm{e}-5$ & 216.81 & 0.022 & $79.9 \%$ \\
\hline
\end{tabular}

The analysis presented for simultaneous coupling and dispersion correction assumes that the local coupling generated by existing triplet roll are fully corrected by the local IR skew quadrupoles. We recommend that these correction be revisited. With properly corrected local coupling, these comparisons between correction algorithm and data will be repeated. With optimum weighting of the coupling versus dispersion, it may be possible to minimize the residual vertical dispersion using vertical dipole correctors, so called dispersion-free-steering (DFS) [12].

With local coupling minimized using existing local skew quads, and global coupling minimized, application of DFS may lead to a truly flat vertical beam orbit (independent of unknown absolute offsets of the beam position monitors).

It is anticipated that simultaneous coupling and dispersion correction will improve accelerator performance and hopefully increase the beam's polarization transmission efficiency during the energy ramp and help preserve polarization during collision for physics studies.

\section{ACKNOWLEDGMENTS}

We would like to thank A. Marusic, V. Ptitsyn, V. Schoefer and C. Zimmer for discussions and interest in this topic. C. Liu would like to thank in particular V. Ptitsyn for invaluable discussions.

[1] C. Montag, L. Ahrens, M. Bai, J. Beebe-Wang, M. Blaskiewicz, J. Brennan, K. Brown, D. Bruno, R. Connolly, T. DOttavio, et al., Proceedings of IPAC10, pg 531 (2010).

[2] R. Assmann, T. Chen, F. Decker, M. Minty, T. Raubenheimer, and R. Siemann, in KEK PROCEEDINGS (NATIONAL LABORATORY FOR HIGH ENERGY PHYSICS, 1995) pp. 463-476.

[3] M. Donald, Y. Cai, H. Shoaee, and G. White, in Particle Accelerator Conference, 1997. Proceedings of the 1997, Vol. 2 (IEEE, 1997) pp. 1454-1456. 
[4] J. Keil and W. Decking, in Proceedings of EPAC (2004).

[5] G. Sahoo, K. Balewski, and W. Decking (EPAC, 2006).

6] F. Pilat, M. Bai, J. Beebe-Wang, J. Cardona, W. Fischer, and V. Ptitsyn, CA/AP 77 (2002).

[7] A. Jain, D. Trbojevic, F. Dell, S. Peggs, P. Wanderer, and J. Wei, RHIC Magnetic Measurements: Definitions and Conventions, Tech. Rep. (RHIC/AP/95, 1996).

[8] Y. Luo, P. Cameron, A. Dellapenna, L. Hoff, A. Marusic, S. Peggs, C. Schultheiss, and R. Jones, Physical Review Special Topics-Accelerators and Beams 9, 124001 (2006).

[9] M. Syphers, Fermilab note Beams-doc-611(2003).

[10] Y. Luo, Sim Track Users Manual (v 1.0), Tech. Rep. (Brookhaven National Laboratory (BNL) Relativistic Heavy Ion Collider, 2010).

[11] F. Pilat, Linear coupling effect of the helical snakes and rotators in RHIC, Tech. Rep. (BNL RHIC/AP/56, 1995).

[12] R. Assmann, P. Raimondi, G. Roy, and J. Wenninger, Phys. Rev. Spec. Top. Accel. Beams 3, 121001 (2000). 